\title{
Die Landesbibliothek unter der Pandemie
}

Eigentlich lagen wir gerade in den letzten Zügen der Arbeiten an unserem Konzept für die Jahre 2020-2025 und hatten die inhaltliche Sortierung von 300.000 Bänden für den Erweiterungsbau gerade in Angriff genommen, da kam Corona. Bevor noch die ersten Verordnungen erlassen wurden, leerten sich die Straßen. Da andernorts aufgrund örtlicher Vorgaben bereits Bibliotheken geschlossen wurden, und es für jeden nur noch eine Frage der Zeit erschien, wann das öffentliche Leben völlig eingestellt würde, kam es zu einer deutlich verstärkten Bestell- und Ausleihtätigkeit. Wir merkten, wie wichtig die Landesbibliothek für unsere Leserinnen und Leser ist. Auch deshalb ist es uns schwer gefallen, zu schließen. Auch wenn wir in den letzten Tagen bereits Hygienevorkehrungen getroffen und Abstandsregelungen eingeführt hatten, ließ es sich nicht vermeiden. Am 17. März 2020 schloss die Landesbibliothek erstmals wieder für mehrere Wochen, 74 Jahre nach ihrer Schließung zwischen dem 13. September 1944 und dem 21. Februar 1946 infolge des verheerenden Luftangriffs auf Stuttgart im Zweiten Weltkrieg.

Wie so viele waren wir nicht vorbereitet. Einen aktuellen Pandemieplan gab es nicht. Und auch Schließen macht Arbeit: Die Öffentlichkeit war zu informieren, viele Nutzeranfragen zu beantworten, die Bestellfunktion des Katalogs und die Mahnfunktion mit Unterstützung der Firma aStec einzufrieren, die Gültigkeit der Ausweise wegen des Zugriffs auf E-Books und E-Journals automatisch zu verlängern, das organisatorische und infrastrukturelle Rückgrat der Bibliothek zu erhalten, die Kolleginnen und Kollegen zu informieren, gesundheitliche Aufklärungsarbeit zu unterstützen, weitere Hygienevorkehrungen zu treffen, gefährdete Mitarbeiter nach Hause zu schicken. Kurz: zunächst sehr viele Aktivitäten unter großer Unsicherheit. Am 25. März wurden dann in der Landesbibliothek als Ziele formuliert:

1. Reduzierung der Risiken für alle,

2. Sicherstellen der Organisation,

3. Steuerung und Aufrechterhaltung der Angebote,
4. breite Nutzung von Home Office,

5. ausgewogene Verteilung von Lasten und Risiken,

6. Schaffung von Stabilität (ggf. über Monate),

7. gute Arbeitsbedingungen,

8. Bearbeitung wichtiger Aufgaben.

Wir hatten uns für 2020 den Abschluss einer Dienstvereinbarung zur Telearbeit vorgenommen. Nun mussten wir aus dem Stand heraus Home Office ermöglichen. Also wurden mit großem Einsatz unserer Digitalen Dienste die erforderlichen Anpassungen vorgenommen und Laptops beschafft und eingerichtet. Die Abteilungs- und Gruppenleiter mussten Arbeiten priorisieren, die auch von zuhause aus erledigt werden konnten. Für manche Aufgaben waren erst noch Kenntnisse zu vermitteln. Und da nicht alles auf einmal möglich war, konnten wir nur schrittweise vorgehen. Zuerst wurde den Kolleginnen und Kollegen Home Office ermöglicht, welche aufgrund von Alter oder Vorerkrankungen zur Risikogruppe gehören (ein Fünftel), unmittelbar ging es mit denen weiter, welche Kinder oder andere Angehörige zu betreuen hatten. Dann kamen die, welche mit besonders gefährdeten Personen zusammenleben und schließlich die, welche besonders lange Anfahrten mit öffentlichen Verkehrsmitteln zu bewältigen haben. Insgesamt machten 57 Kolleginnen und Kollegen von der Möglichkeit des Home Office Gebrauch. Freundlicherweise hat uns die Parkraumgesellschaft Baden-Württemberg $\mathrm{mbH}$ darüber hinaus vorübergehend auch mit Plätzen in benachbarten Tiefgaragen unterstützt.

Ein guter Teil unserer Arbeiten ist an die Papierexemplare gebunden, und unter Einhaltung der Hygieneregeln ist die Arbeit in einer Bibliothek (und noch dazu ohne Publikum) auch zu diesen Zeiten nicht besonders risikobehaftet. Aber dies ist die bequeme Perspektive des Rückblicks. Niemand war gewohnt, mit derartigen Risiken umzugehen oder wusste, wie wirksam die Hygieneregeln gegenüber dem neuartigen Erreger waren. Es gab auch bei uns sehr viele Ängste und unterschiedliche 
Einschätzungen. Es ist wichtig, das normale Leben, die erforderlichen Lern- und Studienbedingungen, die für die Firmen überlebenswichtigen Aufträge an Buchhandlungen, Buchbinder und Restauratoren nicht zum Erliegen zu bringen. Daneben haben wir mehr E-Books für den Fernzugriff lizenziert, als wir es sonst getan hätten. Und wir verschickten Bücher mit der Post und konnten so vielen helfen. Wir mussten wegen gesundheitlicher Gefährdung und fehlenden geeigneten Arbeiten nur sechs von mehr als 180 Mitarbeitern vorübergehend freistellen.

Nach fünf Wochen, am 20. April konnten wir wieder in sehr kleinem Umfang öffnen. Zunächst nur für Studierende und Angehörige der Hochschulen und Doktorandinnen und Doktoranden und für die nur 35 zur Verfügung stehenden Arbeitsplätze unter Angabe von Wichtigkeit und Dringlichkeit der Nutzung des Präsenzbestandes. Die Teilöffnung so zu moderieren, hat sich jedoch nicht bewährt. Es gab berechtigte Kritik von Selbstständigen, die auf die Nutzbarkeit der Bibliothek angewiesen sind, aber angesichts der geringen Zahl der angebotenen Lesesaalplätze waren der Moderation leider Grenzen gesetzt. Zum 11.5. konnte die Anzahl der Leseplätze um 30 auf der Hauptebene der Halle erweitert werden. Das damals auch eingeführte Buchungssystem mit Arbeitsplätzen für eine, zwei, vier oder acht Stunden wird besser akzeptiert. Darüber hinaus konnte damals die Bestellfunktion des Katalogs wieder freigegeben und die Fernleihe wieder aufgenommen werden. Der Zustrom von Besuchern zur Ausleihe ließ sich leichter steuern durch Zuweisung von Öffnungszeiten an Alphabetabschnitte, Einlasskontrolle und die Bereitstellung einer begrenzten Zahl von zu nutzenden Einkaufskörben, zunächst zehn, dann aufgrund entsprechender Regelungen für den Einzelhandel zwanzig. Im Rückblick waren die Schritte zu restriktiv, aber zu diesem Zeitpunkt zählten wir lange zu den wenigen großen Wissenschaftlichen Bibliotheken, die wieder öffneten. Die zeitweilige Beschränkung der Öffnungszeiten auf Mo-Fr 9:00-17:00 Uhr war ärgerlich, angesichts der zu bewältigenden Aufgaben und der Erfordernisse der Pandemie aber leider nicht zu vermeiden.

Home Office war und ist für viele eine Herausforderung. Die regelmäßige Arbeit mit ihren Routinen und zweckmäßigen Einrichtungen sowie der gewohnte Austausch mit Kolleginnen und Kollegen entfallen oder sind erschwert. All dies stabilisierte. Selbstorganisation ist anstrengend, und die kommunikativen Aufwände von Home Office sind ungleich höher. Insbesondere der Wissensfluss und die gemeinsamen Besprechungen litten. Auf die anwesenden Kolleginnen und Kollegen schlug der ungleichmäßige Arbeitsanfall stärker durch, und viele Bitten um Klärung seitens der Abwesenden kamen noch hinzu. Dann mussten die engbesetzten Büros auch noch „entzerrt” werden. Und mit den verstreuten Kolleginnen und Kollegen alles zu organisieren, sich um die Abwesenden besonders zu kümmern, ist eine besondere Herausforderung für die Führungskräfte. Es war und ist noch immer, allein durch das Home Office, für viele eine anstrengende Zeit. Wir werden mit einer Mitarbeiterumfrage den unterschiedlichen Effekten nachgehen, um bei der Einrichtung von regulärer Telearbeit gute Lösungen zu finden. Denn natürlich haben wir auch erlebt, wie fruchtbar und produktiv das ungestörte Arbeiten zuhause sein kann und welch positive Effekte Home Office auf die Vereinbarkeit von Familie und Beruf hat. Manche sind aber aus dem Home Office auch wieder zurückgekehrt, weil es sich für sie nicht bewährte oder weil sie Schwierigkeiten in der Bibliothek sahen.

Daneben hatten wir in diesen Monaten die Vorbereitungen für die Inbetriebnahme des Erweiterungsbaus voranzubringen. Mit sehr großer Konstanz und viel Engagement der Kolleginnen und Kollegen haben wir auch die stilleren Wochen des Lockdowns dafür genutzt, weitere Bestände für die sachlich geordnete Aufstellung im Erweiterungsbau vorzubereiten. Ganz anders die eigentliche Fertigstellung und Inbetriebnahme. Man kann sich kaum vorstellen, wie viele Lösungen und Entscheidungen noch in diesem letzten halben Jahr für die Öffnung des Erweiterungsbaus zu finden waren (und sind). Dies gilt für organisatorische Änderungen, ein weiteres Hygiene-Konzept und neue Angebote im Bibliotheksbetrieb genauso wie für Technik und Ausstattung. Die Nutzung des bisherigen Reservierungssystems zeigt uns, dass wir eigentlich viel mehr Leseplätze bräuchten. Besonders deshalb drängt die Inbetriebnahme des Erweiterungsbaus, weil wir die Anzahl dann um mehr als 100 Plätze erweitern können. Hierfür ist ein leistungsfähigeres Reservierungssystem in Vorbereitung. Gruppenarbeit wird auch dann leider noch nicht möglich sein. 
Von den Firmen ist in diesen Wochen viel geleistet worden, immer unter der Unsicherheit, ob und wie es weitergeht. Die Erreichbarkeit von Behörden, die großen Unsicherheiten der Fertigstellungstermine und die langwierigere Entscheidungsfindung mit mehreren Partnern waren eher ein Problem. Neben der großflächigen Beschädigung der Isolierung unseres Vorplatzes hat auch die Pandemie einen deutlichen Anteil daran, dass die Fertigstellung länger braucht und Vieles nun in sehr kurzer Zeit zu bewältigen ist. Wenn wir unsere Öffnungszeiten nicht auf den Stand vor Corona zurücksetzen können, dann liegt das also keineswegs an der "Faulheit der Beamten im bequemen Home Office“, wie es uns in manchen Zuschriften vorgeworfen wird.

Bei der Erstellung, Umsetzung und wiederholten Überarbeitung des Hygienekonzeptes hat der enge Austausch zwischen den Leitungen der großen Wissenschaftlichen Bibliotheken des Landes geholfen. Aber wir sind froh, viel schneller und spezifischer reagieren zu können, als dies in universitären Gesamtkonzepten möglich ist. Außerdem haben unser betriebsärztlicher Dienst und unsere Fachkraft für Arbeitssicherheit Fachkenntnisse und eine nüchterne Außenperspektive eingebracht. Die ersten Konzepte waren von ähnlichen Unsicherheiten geprägt wie die ersten Corona-Verordnungen. Diese Unsicherheiten erhöhten sich durch ihre widersprüchlichen Umsetzungen in verschiedenen Einrichtungen. Besonders aufwendig waren die Änderungen in Möblierung und technischer Ausstattung sowie die Einführung von Reservierungslösungen. Und stellen Sie sich vor: Die Hölderlin-Ausstellung mussten wir wegen zunächst nicht fertiggestellter Räume und der Pandemie drei Mal planen. Sowohl durch die Pandemie als auch durch Unwägbarkeiten der Fertigstellung des Erweiterungsbaus kamen viele unvorhersehbare Kosten zusammen, und Sparen bindet deutlich mehr Kräfte als Ausgeben.

In Anbetracht der zeitweiligen niedrigen Infektionszahlen und der in greifbarer Nähe erscheinenden Inbetriebnahme des Erweiterungsbaus entstanden zwischenzeitlich Phasen, in welchen es schwerfiel, die Befolgung der Hygiene-Standards konsequent durchzuhalten. Natürlich wurde man zwischenzeitig der Problematik überdrüssig. Angesichts erneut steigender Infektionszahlen wird nun wieder umsichtiger gearbeitet und wir bemühen uns, dass das regelmäßige Lüften der nicht klimatisierten Bereiche zur Routine wird. Die Pandemie wird uns wohl noch lange begleiten. Wir hoffen dennoch, die Öffnung des Neubaus mit Gästen in heiterer Weise begehen zu können. Das wird kein Fest mit Buffet und ungezwungenem Beisammensein, wie man es aus früheren Zeiten kennt. Aber es soll viel zu sehen und zu hören geben und ausreichend Platz für gute Begegnungen und Austausch in kleineren Kreisen. Im Oktober können wir dann außerdem endlich die Hölderlin-Ausstellung eröffnen und wieder Veranstaltungen anbieten. Alles kann nur nach Voranmeldung besucht werden. Hoffentlich setzen die Infektionsraten den Rahmen nicht zu eng.

\section{Rupert Schaab}

